\title{
Linking niche number, environmental filter, conditional neutrality and space size to understand species richness through environmental gradient per unit space
}

\author{
Qingshi Zhou ${ }^{1}$, Yang $\mathrm{Gao}^{2}$, Zong Cheng $\mathrm{Ma}^{3}$, and Long Tang ${ }^{4}$ \\ ${ }^{1}$ Xian Jiaotong University \\ ${ }^{2}$ Xi'an University of Technology \\ ${ }^{3}$ Affiliation not available \\ ${ }^{4}$ Xi'an Jiaotong University
}

May 5, 2020

\begin{abstract}
Although numerous studies have been conducted on niche and neutral theories to learn the drivers of species richness, few of them have demonstrated how to eliminate the influences of unlimited species numbers and absolute species equivalences which are contrary to many observations, and how to link space size with those drivers posited by the two theories. Here we present the environmental gradient per unit space metric that influences niche number, positively correlates with environmental filter and dispersal limitation and negatively correlates with space size occupied by each niche. This metric is incorporated with stochastic abundance and migration and equivalently average birth, death and dispersal of species. The simulation result of a model is consistent with the observation that a unimodal algal richness-water environmental gradient per unit space relationship. Therefore, the environmental gradient per unit space connecting deterministic and stochastic processes is an importantly measurable driver of species richness.
\end{abstract}

\section{Introduction}

An important role of ecologists is to explain and predict spatial differences in species richness. To this end, niche and neutral theories have been developed (Abrams 1983; Hubbell 2005; Schwilk \& Ackerly 2005; Gravel et al . 2006; Kadmon \& Allouche 2007; Levine \& HilleRisLambers 2009; Hart et al . 2017). However, both two theories do not predict the effects of space size that can influence population size and thus population extinction risk on species richness, and the potential prediction that unlimited species number of niche theories and the prediction that absolute species equivalence of neutral theories are contrary to numerous observations (Abrams 1983; Rohde 1992; Rahbek 1995; Hubbell 2005; Tilman 2005 Nogues-Bravo et al . 2008; Mellard et al . 2012; Mandal et al . 2018). Most studies that have been conducted to reconcile the two theories are focused on the variously contradictory predictions of the two theories, such as differences and equivalences of species, competitive exclusions and stochastic extinction, and environmental filters and dispersal limitations (Schwilk \& Ackerly 2005; Tilman 2005; Gravel et al . 2006; Kadmon \& Allouche 2007; Peng et al . 2016; Mitchellet al . 2019). As a result, there are few comprehensive insights into species richness, and such insights should link space size with deterministic and stochastic processes and overcome the shortcomings that unlimited species numbers and absolute equivalences.

Here, the environmental gradient per unit space (EGUS) metric, also termed the environmental spatial change rate, is presented. The EGUS of an area positively correlates with the environmental gradient in the area. When each environmental gradient is regarded as an optimal niche, the EGUS in an area consequently 
limits the number of species in an area. The EGUS should positively correlate with environmental filter and dispersal limitation and negatively correlate with space size which is occupied by an optimal niche and therefore is regarded as environmental capacity of a species. Further, this metric is incorporated with stochastic abundance change of each species, stochastic migration and equivalently average birth, death and dispersal of species along environmental gradients. In this way, the space size, differences and equivalences of species, and deterministic and stochastic processes are integrated through the EGUS.

The EGUS model is developed to demonstrate how such integrations influence species richness. An investigation of the relationship between algal richness and water EGUS in a lake and a river is conducted. If the simulation results are consistent with the observations, the EGUS should be an importantly measurable driver of species richness.

\section{Methods}

\section{Model}

To involve the drivers as comprehensive as possible, four perspectives form the heart of EGUS model. According to niche theories and observations, first, each species should have a unique optimal niche. The regular spacing of niches wherein niche differences exceed a threshold, as in limiting similarity created by competitive exclusion (Abrams 1983; Schwilk \& Ackerly 2005; Gravel et al . 2006), supports the coexistence of competitive winners which definitively and stably contribute to the richness. Immigrant niches can exist in the intervals among native species niches and can be created after the competitive exclusion of natives (Schwilk \& Ackerly 2005; Gravelet al . 2006). In this scenario, the regular spacing of niches should be the regular spacing of optimal niches. If a species is competitively excluded in the spaces with its optimal niche, the species need not to be considered because it only can trigger a fluctuation of richness. Second, most species perform well in moderate environments (Rohde 1992; Rahbek 1995; Nogues-Bravo et al . 2008; Mellardet al . 2012; Mandal et al . 2018), and optimal niches should gather at the moderate environmental range and should normally distribute along stressful level. According to neutral theories and some observations (Hubbell 2005; Gravel et al . 2006; Mellard et al . 2012; Mandal et al . 2018), third, the averages of values of birth rates, death rates, dispersal breaths and environmental capacities of species are equivalent among different areas of an ecosystem and species should be equivalent in niche breaths. Forth, the individual birth, death and dispersal of a species should be random and thereby the abundance should be random.

An area with a high EGUS exhibits a large environmental range, a high environmental gradient, a low space size occupied by each niche and a low environmental capacity of each species. Population extinction risks increase with decreasing population sizes caused by declining environmental capacities (Annette 2005; He 2012). Then

$E_{\text {range }}=E_{\max }-E_{\min }(1)$

$E_{k}=E_{\text {min }}+\frac{k}{K} E_{\text {range }}(2)$

where $E_{r a n g e}$ is the environmental range of a random area in an ecosystem. $E_{\min }$ and $E_{\max }$ are the environmental minimum and maximum of the area, respectively. The area has $K$ sections and $K$ is a linear function of $E_{\text {range }}$. A section has an environmental value and thereby is an optimal niche. $E_{k}$ is the environmental value for a random section $k$. J sites distribute among $K$ sections and section $k$ has $J_{k}$ sites. Each site is occupied by one individual and the numbers of sites are same among sections due to the same environmental capacity.

Because of the randomness of arrival time and abundance of species at initial stages of community formations, the space with the optimal niche of a species is not necessary to be totally occupied by the individuals of this species. Thus, algal individuals should often randomly die due to deterministic processes such as the mismatch between species preferences and environments and competitive exclusions and stochastically emigrate. The abundance of each species is random, and therefore the decreases of richness or stabilization after individual death or emigration have been considered. Further, if vacant sites created by random death and emigration in section $k$ are occupied by immigrants from other sections or other areas but not native 
offspring in section $k$, the richness in the area including section $k$ could profoundly change. Then based on previous studies (Schwilk \& Ackerly 2005; Kadmon \& Allouche 2007; Allouchea et al 2012; Bar-Massada 2015), the species composition in section $k$ over time is:

$N_{k, t+1}=\left\lfloor(1-m) N_{k, t}\right\rfloor+B_{k, t}+I_{k, t}(3)$

where $N_{k, t}$ and $N_{k, t+1}$ are the abundances of all species in section $k$ at time $t$ and $t+1$, respectively. $m$ is the mortality and emigrant rate, and therefore is the proportion of vacant sites to the total sites in section $k$ at time $t$. Because the species randomly distributes among areas at initial community formation, the averages of death and emigrant rates of species are equivalent among sections, $m$ is same among sections. The term in the bracket is a floor function, and the abundance is integer. $B_{k, t}$ is the abundance of native offspring from time $t$ to time $t+1 . I_{k, t}$ is the abundance of immigrant species from time $t$ to time $t+1$.

The potential proportion of vacant sites in section $k$ being occupied by immigrants is:

$p_{k}=\frac{c}{c+r\left(\frac{J_{k}}{J}\right)}(4)$

where $c$ is immigration rate and thus is the proportion of immigrant abundance in total abundance. Because random and equivalent dispersal, $c$ is same among sections. $r$ is the birth rates of native species in section $k$ and thus is the proportion of abundance of all new offspring in the abundance of all natives in section $k$. Again, because the species randomly distributes, the average probabilities of native births are same among sections, $r$ is same.

Dispersal limitation and environmental filter are important stochastic and deterministic processes posited by neutral and niche theories (Hubbell 2005; Gravel et al . 2006; Levine \& HilleRisLambers 2009), respectively. Then the survival rate of immigrants in section $k$ is:

$R_{s, k}=\frac{\left(\sum_{x=1}^{K} D_{x, k} N_{s, x}+\sum_{l=1}^{L} D_{l, k} N_{s, l}\right) F_{s, k}}{\sum_{s=1}^{S}\left(\sum_{x=1}^{K} D_{x, k} N_{s, x}+\sum_{l=1}^{L} D_{l, k} N_{s, l}\right) F_{s, k}}(5)$

where $D_{x, k}$ is the possibility of the arrival of a random species $s$ from section $x$ to section $k$, and $x$ and $k$ are in the same area. Because the focus is local community and algal movability is high, algal individuals can spread freely among sections in an area and the value of $D_{x, k}$ is set as $100 \% . N_{s, x}$ is the abundance of species $s$ in section $x . L$ is the number of adjacent areas wherein species may be able to arrive at the section $k$ , $D_{l, k}$ is the possibility of the arrival of species from another area $l$ to section $k$ (as calculated by equation 6 ). $N_{s, l}$ is the abundance of speciess in area $l . F_{s, k}$ is the tolerance of species $s$ to the environmental value of section $k$ (as calculated by equation 7 ). $S$ is the total species richness in the simulated system.

Dispersal limitation: the possibility of arrival of an immigrant from another area $l$ to section $k$ is:

$D_{l, k}=e^{\frac{-(l-k)^{2}}{2 \theta_{s}^{2}}}(6)$

where $\vartheta_{\mathrm{S}}$ is the dispersal breadth of species $s$ and is equivalent among species.

Environmental filter: species tolerance to the environment of section $k$ is :

$F_{s, k}=e^{\frac{-\left(e_{s}-E_{k}\right)^{2}}{2 \sigma_{s}^{2}}}(7)$

where $e_{s}$ is the niche optimum of species sand is different among species. is the niche breadth of species $s$ and is equivalent among species.

$J$ was set as 300, and thus, the abundance of species in an area was 300 . The species richness $(S)$ in the simulated ecosystem was set as 300. $K=100 \times E_{\text {range }}$. All species had a same birth rate $(r)$ of 10 , a same mortality rate $(m)$ of 0.25 , a same immigration rate $(c)$ of 0.2 , and a same niche width () of 0.4 . Moderate environmental values were set as between 0 and 2, with values between 0 and 1 containing the highest number of optimal niche. 
Two simulation methods were used. In the first method, the areas were divided into heterogeneous, transitional, and homogeneous according to high EGUS, middle EGUS, and low EGUS. The environmental ranges $E_{\text {rang }} \mathrm{s}$ of these areas were $60 \%, 40 \%$, and $20 \%$ of the entire environmental range (0.01-10) of the simulated ecosystem, and thus, the values of the range were 6,4 and 2 , respectively. The environmental stress level of each area was varied (Table 1). In the second method, the $E_{\text {rang }}$ exhibited seven levels including 0.01 and 1 to 6 , and the environmental minimum $E_{\min }$ of each $E_{\text {rang }}$ was a random value between 0 and 3. Thus, the simulations should cover the possible combinations of environmental gradient levels and stressful levels and thereby should cover almost all natural cases such as areas exhibit a high stress with a large environmental range and thus a high environmental gradient, areas exhibit a high stress with a narrow range and thus a low gradient, and areas exhibit a low stress with various ranges and gradients.

The species in each area were randomly sampled when the simulations started to run. The model was run over 1,000 time steps, such that species richness stabilized by the end of the run. The replications of the first and second method were 10 and 30, respectively. ANOVA followed by Tukey's HSD test was employed to analyze the effects of environmental stress levels and environmental range on richness in the results of the first method. Negative binomial regression was employed to analyze the relationship between EGUS and species richness in the results of the second method. The significance was 0.05 . The model and results were evaluated in the R programming system (Seattle, WA: MathSoft, Inc).

\section{Investigation}

The Ankang Reservoir (32³6'6.11" N, 108deg53'21.26" E), also referred to as Yinghu, is in the upper reaches of the Hanjiang River, which is the largest tributary to the Yangtze River. The reservoir area is $38,625 \mathrm{~km}^{2}$ and the total capacity is $25.8 \times 10^{8} \mathrm{~m}^{3}$, with a multiyear mean discharge of 190 million $\mathrm{m}^{3}$. The multiyear mean water temperature is $14-16 \mathrm{degC}$, and the multiyear mean rainfall is $800-1,100 \mathrm{~mm}$.

Water samples were collected in mid-July from 17 sample locations in the reservoir that were 1 to $33 \mathrm{~km}$ from the dam, with $2 \mathrm{~km}$ between neighboring sample locations. Samples were taken from five depth levels $(0.5,2.5,5,10$, and $20 \mathrm{~m})$ at each location. Nine sampling locations were downstream in the river, $1 \mathrm{~km}$ to $17 \mathrm{~km}$ from the dam, with $2 \mathrm{~km}$ between neighboring locations. Because water depth in the river was less than $4 \mathrm{~m}$, the water samples were taken at 0.5 and $2.5 \mathrm{~m}$ depth levels at each location. One liter of water was fixed with $1 \%$ Logul's solution to measure the diversity and abundance of algae, while $500 \mathrm{ml}$ of water was refrigerated at $4 \mathrm{degC}$ for subsequent nutrient analyses. Each sample was replicated in triplicate.

Temperature, $\mathrm{pH}$, dissolved oxygen, and salinity were measured with a portable water quality analyzer (Hydrolab DS5, HACH, America). Total nitrogen and total phosphorus were measured with a spectrophotometer (DR6000, HACH, America). One liter of water was used for algal identification analysis after letting settle for $48 \mathrm{~h}$. The upper $950 \mathrm{ml}$ of water was then removed and $0.1 \mathrm{ml}$ of water was taken from the remaining $50 \mathrm{ml}$ for microscopic identification of algal abundances and species.

Principal component analysis (PCA) was used to analyze the variation in the six measured environmental variables. The first two axes explained $82.67 \%$ of the variation in the environmental parameters in the reservoir samples $(\mathrm{PC} 1=58.89 \%$ and $\mathrm{PC} 2=23.78 \%)$ and $81.10 \%$ of the variation in the variables in the downstream river samples $(\mathrm{PC} 1=54.46 \%$ and $\mathrm{PC} 2=26.65 \%)$. The percentage of explained variance by each axis (i.e., PC1 and PC2 values) was taken as its weight of the PCA score for each axis. The value of the PCA score for each axis was multiplied by its weight, and the two products were added to determine the environmental value at each site. The environmental values for the reservoir and downstream river sites were then calculated.

The local Moran $I\left(I_{i}\right)$ values of the reservoir and downstream sites were calculated to measure the EGUS of water as follows (Massicotte et al . 2015):

$I_{i}=\frac{z_{i}-z}{\sigma^{2}} \sum_{j=1, j \neq i}^{n}\left[W_{\mathrm{ij}}\left(z_{j}-z\right)\right]$

where $z_{i}$ and $z_{j}$ are the environmental values for sites $i$ and $j$, respectively. $z$ is the environmental average 
value of all the sites and $n$ is the number of sites. $\sigma^{2}$ is the variance of $z$ and $W_{i j}$ is a distance weighting factor between site $i$ and site $j$ that is the inverse of the distance. A lower Moran's $I$ value of a site represent a more significant difference between the site and adjacent sites. I values were calculated in Stata 12.0 (STATA, America).

Negative binomial regression test was used to evaluate the relationship between each Moran $I$ value and algal richness in the reservoir and downstream river sites. A significance level was set as 0.05 . These analyses were performed in the SPSS 19.0 software package (IBM, America).

\section{Results}

When the areas in an ecosystem are sorted into the heterogeneous, transitional and homogeneous according to the low, middle and high EGUS value, the results of simulation showed that species richness is often the highest in the area with a middle EGUS value, followed by those in the area with a high value, and lastly, in the area with a low value. The area with a high EGUS value is the highest only when the environmental stress levels are very high in these areas (Table 1). The second simulation method revealed a unimodal relationship between species richness and EGUS (Fig. 1).

The investigation also showed a unimodal algal richness-water EGUS value relationship (Fig. 2). After the locations were matched with local Moran $I\left(I_{i}\right)$ values, the results showed that the EGUS of water in the middle layer of the lake is the highest, followed by that of the shallow layer, and lastly, that of the deeper layer. The algal richness of the middle and deeper layers decrease by about $5 \%$ and $12 \%$ compared to that of the shallow layer, respectively. The EGUS of the river section closest to a city is the highest, followed by that of the section closest to a dam, and lastly, that of the section passing by the city. The algal richness in the river section closest to the city and the section passing by the city decrease by about $9 \%$ and $26 \%$ compared to that of the section closest to the dam, respectively.

\section{Discussion}

The simulation results are consistence to the observations, which demonstrates that the EGUS is a measurable driver of species richness. Each environmental gradient is regarded as an optimal niche, a low EGUS means a low number of optimal niches. Nevertheless, environmental gradients exceeding the range in which optimal niches concentrate should only provide optimal niches for few species that prefer stressful environments and many environments are unavailable for species. Thus, considerable increases in EGUS could not significantly increase the numbers of species; further, such increases elevate abiotic filter and dispersal limitation in the way that migrants move towards spaces exhibiting their optimal niches, thereby reducing immigration success, and such increases decrease the sizes of the space occupied by each optimal niche. Population sizes are small within narrow spaces and both extinction risks and dispersal limitation are high (Annette 2005; He 2012). The optimal niche number is relatively high in the areas with middle EGUS except that such areas exhibit a high environmental stress levels and those negative effects are relatively little. In this way, both the simulations and the observations can show a unimodal algal richness-EGUS relationship.

The consistency between the simulations and observations imply algal species probably exhibit a difference of environmental preference and a conditional equivalence. Species equivalence has been criticized because a species often performs differently in different environments and different species in same environments exhibit different performances (Rohde 1992; Rahbek 1995; Nogues-Bravo et al . 2008; Mellardet al . 2012; Mandal et al . 2018). According to these observations, species equivalence should be understood as a conditional equivalence and the conditions should be same type species and average performances of these species. The changes of performances of a species along environmental gradients probably cause that the averages of performances along a relatively wide environmental range are approximately same among same type species such as among algae, among $K$-strategy species and among $r$-strategy species. Thus, again, when same type species randomly distributes among environments, the averages of births, deaths, migrations, tolerances, dispersal deaths, and environmental capacities of species are equivalent among environmental levels. The results also imply that deterministic competitions and environmental filters, and dispersal limitations and stochastic abundances should influence algal richness, supporting our predictions. 
In addition to environmental range, fragmentation levels involving habitat loss and habitat apart and the composition of factors like nitrogen availability ratios and light spectra are often measurements of the environmental property (Fahrig 2003; Schuler et al . 2017; Mandal et al . 2018), but spatial scales are subjective. We can employ the EGUS to connect fragmentation levels and compositions of factors in each sampling position to sort the areas independently of spatial scale and relative position of areas. Such classification is objective and can indicate community boundary. Further, the EGUS can profoundly influence immigrant success, population extinction and niche availability. Therefore, the EGUS is an importantly measurable driver of species richness, and the theory provides a potential understanding and prediction of terrestrial plant richness and invasive plant spread when the migrants in the study are regarded as propagules, especially, seeds of annual plants.

\section{Acknowledgements}

Funding : The National Natural Science Foundation of China (No. 31670548, No. 31872032 and NO. 31500340) and the Fundamental Research Funds for Central Universities.

\section{References and Notes}

Abrams, P. (1983). The theory of limiting similarity. Ann. Rev. Ecol. Syst. , 14, 359-376.

Allouchea, O., Kalyuzhnya, M., Moreno-Ruedab, G., Pizarrob, M. \& Kadmona, R. (2012). Areaheterogeneity tradeoff and the diversity of ecological communities. Proc. Natl. Acad. Sci. U.S.A., 109, $17495-17500$.

Annette, K. (2005). Reduced reproductive success and offspring survival in fragmented populations of the forest herb Phyteuma spicatum .J. Ecol. , 93, 1226-1237.

Bar-Massada, A. (2015). Immigration rates and species niche characteristics affect the relationship between species richness and habitat heterogeneity in modeled meta-communities. Peer $J$, 3, e832 [Peer J-the Journal of Life and Environmental Sciences]

Fahrig, L. (2003). Effects of habitat fragmentation on biodiversity.Annu. Rev. Ecol. Evol. Syst. , 34, $487-515$.

Gravel, D., Canham, C.D., Beaudet, M. \& Messier, C. (2006). Reconciling niche and neutrality: the continuum hypothesis. Ecol. Lett. , 9, 399-409.

Hart, S.P., Usinowicz, J. \& Levine, J.M. (2017). The spatial scales of species coexistence. Nat. Ecol. Evol. , 1, 1066-1073.

He, F.L. (2012). Area-based assessment of extinction risk.Ecology , 93, 974-980.

Hubbell, S.P. (2005). Neutral theory in community ecology and the hypothesis of functional equivalence. Funct. Ecol. , 19, 166-172.

Kadmon, R. \& Allouche, O. (2007). Integrating the effects of area, isolation, and habitat heterogeneity on species diversity: a unification of island biogeography and niche theory. Am. Nat. , 170, 443-454.

Levine, J.M. \& HilleRisLambers, J. (2009). The importance of niches for the maintenance of species diversity. Nature , 461, 254-257.

Mandal, S., Shurin, J.B., Efroymson, R.A. \& Mathews, T.J. (2018). Heterogeneity in nitrogen sources enhances productivity and nutrient use efficiency in algal polycultures. Environ. Sci. Technol. , 52, 37693776 .

Mellard, J.P., Yoshiyama, K., Klausmeier, C.A. \& Litchman, E. (2012). Experimental test of phytoplankton competition for nutrients and light in poorly mixed water columns. Ecol. Monogr. , 82, 239-256. 
Mitchell, E.G., Harris, S., Kenchington, C.G., Vixseboxse, P., Roberts, L., Clark, C., Dennis, A., Liu, A.G. \& Wilby, P.R. (2019). The importance of neutral over niche processes in structuring Ediacaran early animal communities. Ecol . Lett ., 22: 2028-2038.

Nogues-Bravo, D., Araujo, M.B., Romdal, T. \& Rahbek, C. (2008). Scale effects and human impact on the elevational species richness gradients.Nature , 453, 216-219.

Peng, C., Jun, T.W., Hang, W.H., Yuan, M Z., Yuan, G., Ju, P.S. \& Ji, Z.H. (2016). Environmental filtering process has more important roles than dispersal limitation in shaping large-scale prokaryotic beta diversity patterns of grassland soils. Microb. Ecol ., 72, 221-230.

Rahbek, C. (1995). The elevational gradient of species richness-a uniform pattern. Ecography , 18, 200-205.

Rohde, K. (1992). Latitudinal gradients in species diversity: the search for the primary cause. Oikos , 65, $514-527$.

Schuler, M.S., Chase, J.M. \& Knight, T.M. (2017). Habitat size modulates the influence of heterogeneity on species richness patterns in a model zooplankton community. Ecology , 98, 1651-1659.

Schwilk, D.W. \& Ackerly, D.D. (2005). Limiting similarity and functional diversity along environmental gradients. Ecol. Lett. , 8, 272-281.

Tilman, D. (2005). Niche tradeoffs, neutrality, and community structure: A stochastic theory of resource competition, invasion, and community assembly. Proc. Natl. Acad. Sci. USA, 101, 10854-10861.

Table 1. Modeled richness corresponding to environmental stress levels and EGUSs

\begin{tabular}{llllll}
\hline Environmental stress level & Heterogeneous area & Heterogeneous area & Heterogeneous area & Transitional area & Transitio \\
\hline & $E_{\min }$ & $E_{\max }$ & Richness & $E_{\min }$ & $E_{\max }$ \\
All optima niche & 0 & 6 & $108.4 \pm 4.7 \mathrm{~b}$ & 0 & 4 \\
Half optimal niche & 1 & 7 & $91.3 \pm 4.3 \mathrm{~b}$ & 1 & 5 \\
No optimal niche & 3 & 9 & $51.4 \pm 3.0 \mathrm{a}$ & 3 & 7 \\
Highest stress & 4 & 10 & $34.4 \pm 3.8 \mathrm{a}$ & 6 & 10 \\
\hline
\end{tabular}

Note: $E_{\min }$ and $E_{\max }$ are the environmental minimum and maximum of an area, respectively. Lowercase letters in a line indicated the significant differences in species richness. 


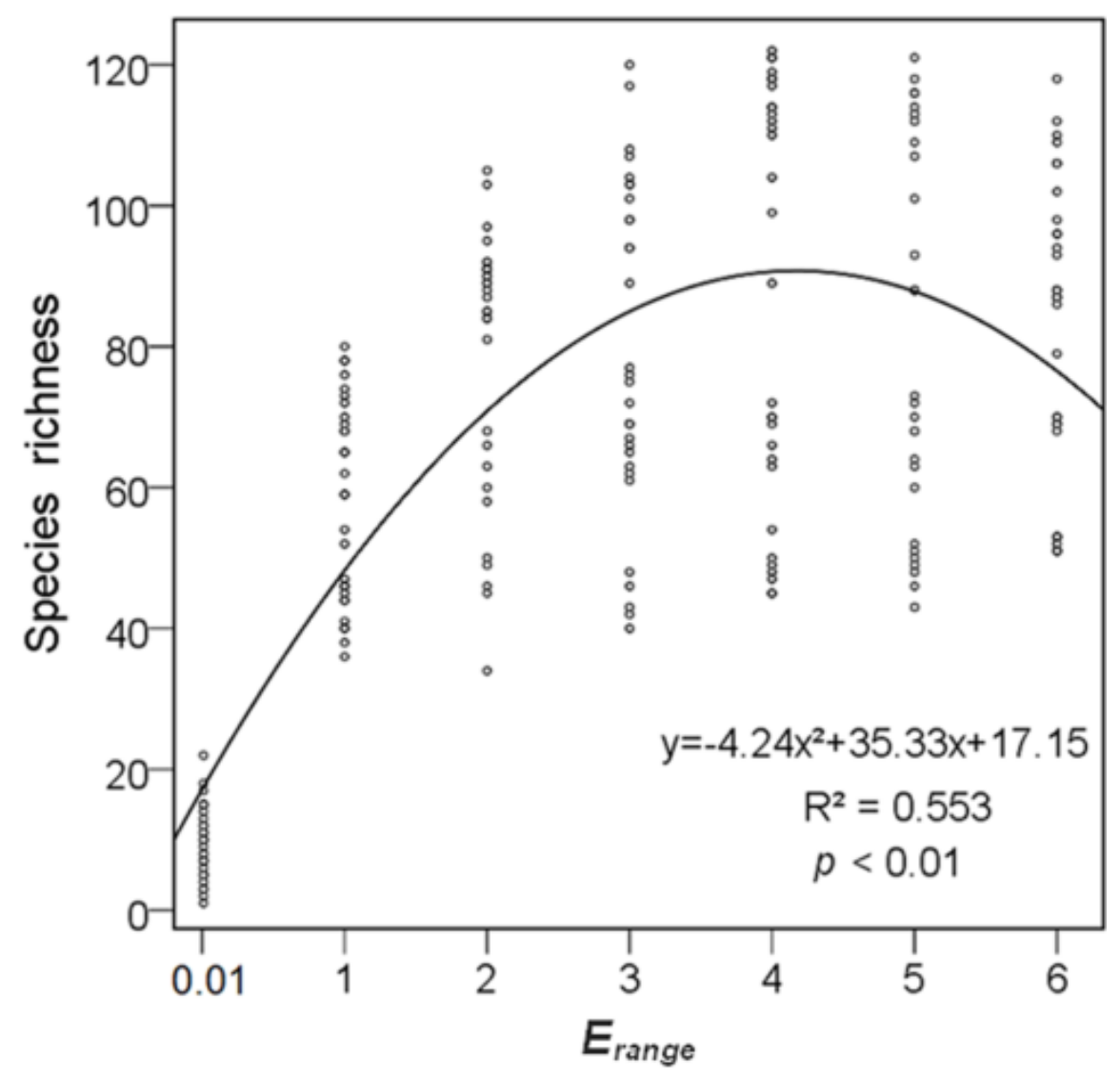

Environmental gradient per unit space increase

Fig. 1. A simulated species richness-EGUS relationship.

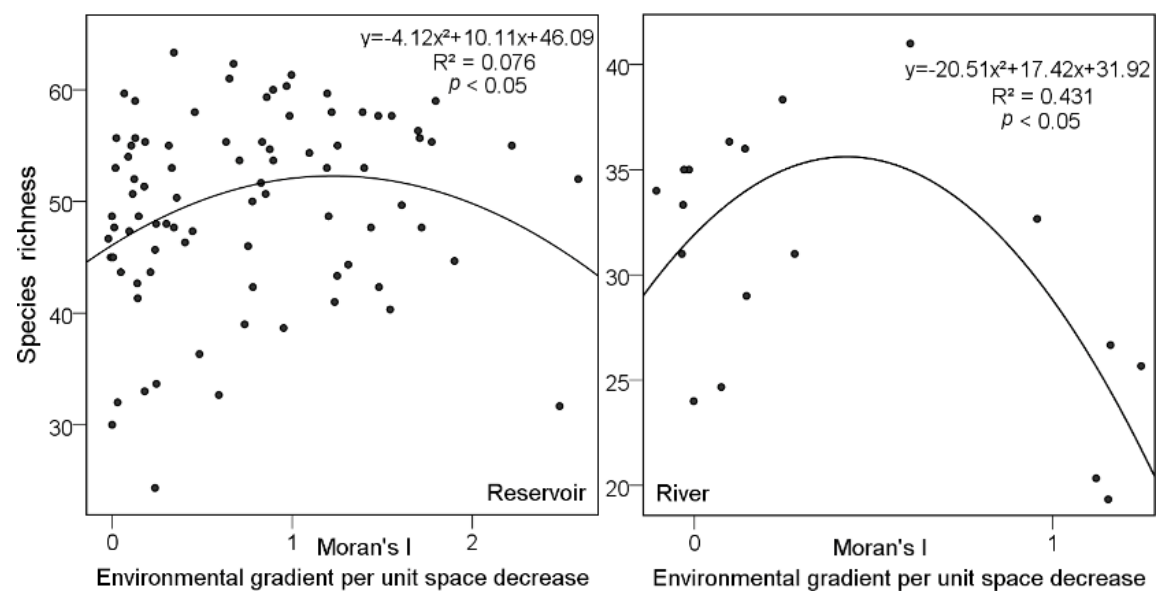

Fig. 2. Unimodal relationships between algal richness and environmental gradient per unit space of water. 